\title{
Antibiotic Resistant Bacterial Pathogens Associated with Blood Stream Infections and Urinary Tract Infections among Intensive Care Unit Patients
}

\author{
Shriram Dorairaj Gunasekaran ${ }^{1}$ (D) , Godfred Antony Menezes ${ }^{2 *}$ (D), Rezmitha \\ Zulfihar Nazeem ${ }^{1}$ (D) Mohammad Obada Sultan ${ }^{1}$ (D) Tejaswini Archarya $^{1}$ (D), \\ Shaista Khadim ${ }^{1}$ (D), Resshme Kannan Sudha ${ }^{1}$ (D) , Harshita Janardhan ${ }^{1}$ (D), \\ Mrinali Sanjay Bhatia ${ }^{1} \mathbb{D}$, Farzeen Fathima ${ }^{1} \mathbb{D}$, Khulood AlKhateri ${ }^{3} \mathbb{D}$, Michael \\ Magaogao $^{1}$ (D) Sara Babiker Ali $^{3}$ (D) , Hanadi Elias Hassan ${ }^{3}$ (D), Essam El-Din Mahran \\ Aly $^{3}$ and Azan Saleh Al Saadi ${ }^{1,3}$ (D) \\ ${ }^{1}$ RAK College of Medical Sciences (RAKCOMS), RAK Medical \& Health Sciences University (RAKMHSU), \\ Ras Al Khaimah, UAE. \\ ${ }^{2}$ Associate Professor \& Clinical Microbiologist, RAK College of Medical Sciences (RAKCOMS), \\ RAK Medical \& Health Sciences University (RAKMHSU), Ras Al Khaimah, UAE. \\ ${ }^{3}$ Saqr Hospital, Ras Al Khaimah, UAE.
}

\begin{abstract}
Blood Stream Infection (BSI) and Urinary Tract Infection (UTI) being leading causes of morbidity and mortality represent a common complication among critically ill patients. During the last decade, clinicians have observed a rising occurrence of BSIs due to bacterial resistance. Likewise, catheterassociated UTI is a main cause of morbidity and mortality affecting all age groups. Coliforms happen to be the prominent pathogens among our ICU admitted patients. It was alarming to notice $\mathbf{4 2 . 9 \%}$ resistance to tigecycline among $K$. pneumoniae isolated from blood. $K$. pneumoniae isolates cultured from urine of ICU patients uniformly displayed $75 \%$ resistance to ciprofloxacin, ceftriaxone, cefoxitin and cefepime. Interestingly, it is of respite to observe $85.7 \% \mathrm{~K}$. pneumoniae isolated from blood and $75 \% K$. pneumoniae isolated from urine being susceptible to a conventional antibiotic, gentamicin. Escherichia coli isolated from urine were $100 \%$ susceptible to carbapenems and $91.75 \%$ were susceptible to tigecycline. Overall, $\mathbf{9 0 \%}$ of Pseudomonas aeruginosa were susceptible to nitrofurantoin. The rapid spread of these MDR pathogens demands for national and regional guidelines. Policies to treat ICU related infections in UAE should be designed based on local microbiological data and resistance profiles of pathogens.
\end{abstract}

Keywords: Antimicrobial Resistance, pathogens, Blood Stream Infections, Urinary Tract Infections, Intensive Care Unit Patients

*Correspondence: godfred@rakmhsu.ac.ae; : +971 543266244

(Received: January 27, 2020; accepted: July 16, 2020)

Citation: Gunasekaran SD, Menezes GA, Nazeem RZ, et al. Antibiotic Resistant Bacterial Pathogens Associated with Blood Stream Infections and Urinary Tract Infections among Intensive Care Unit Patients. J Pure App/ Microbiol. 2020;14(3):1737-1748. doi: 10.22207/JPAM.14.3.12

(C) The Author(s) 2020. Open Access. This article is distributed under the terms of the Creative Commons Attribution 4.0 International License which permits unrestricted use, sharing, distribution, and reproduction in any medium, provided you give appropriate credit to the original author(s) and the source, provide a link to the Creative Commons license, and indicate if changes were made. 


\section{INTRODUCTION}

In spite of controlling many risk factors, there is higher risk of infections in intensive care units (ICUs) compared with regular wards. In comparison to pneumonia and wound infections, BSI and UTI have a higher incidence in ICUs compared with other wards. Patients should be managed in ICUs for an optimal period that is necessary for their care ${ }^{1-4}$. Effective and prompt antibiotic therapy is necessary in order to improve clinical outcomes and reduce mortality. Nevertheless, due to worldwide spread of multidrug resistant (MDR) organisms, the choice of empiric antibiotic regimen is often challenging. We need to have new therapeutic challenges to minimize the risk of resistance selection in turn to improve the effectiveness of antibiotic treatment ${ }^{5}$. BSIs form leading infections among critically ill patients. The case-fatality rate related to BSIs in patients admitted to ICUs is very high (35-50\%). Antimicrobial therapy is the cornerstone for treating BSIs, which are solely responsible for leading into severe sepsis and septic shock. During the last decade, clinicians have observed a rising occurrence of BSls by bacteria resistant to commonly used antimicrobials ${ }^{6,7}$. A MDR microorganism is resistant to at least one agent in three or more antimicrobial classes. Extensive drug-resistant (XDR) microorganisms is susceptible to only one or two antimicrobial classes, while pan-DR (PDR) isolates are resistant to all agents from all antimicrobial classes ${ }^{8}$. Emergence of antibiotic resistance is a global public health problem $^{9,10}$. Likewise, catheter-associated UTI is a main cause of morbidity and mortality affecting all age groups ${ }^{11}$. Bacteriuria or candiduria is nearly unavoidable in half of the patients on indwelling urinary catheter for more than five days. Patients with asymptomatic bacteriuria harbor major pool of antibiotic resistant pathogens in hospitals ${ }^{12}$. With this background in mind, we designed this study with the following aim: to study the prevalence of BSIs and UTIs caused by antibiotic resistant bacterial pathogens among ICU Patients. The objectives of the study were to analyze the types of bacterial isolates cultured from blood and urine samples of ICU patients; to determine the bacterial drug resistance profile and to understand the bacterial resistance pattern among BSI and UTI causing pathogens from ICU in past 2 years.

\section{MATERIALS AND METHODS}

It is a cross-sectional and retrospective study. The bacterial isolates from patients with BSIs and UTIs, who were admitted in ICUs of a tertiary care hospital (ICUs of Saqr Hospital, Ras Al Khaimah, UAE) of a 2 years period (2016 and 2017) were included. Institutional Ethical Clearance and regional ethical clearance (2018) approval has been obtained for the study.

\section{Bacterial isolation, identification and antibiotic} susceptibility testing

Both Gram positive and Gram-negative bacteria isolated from blood and urine of patients suffering from BSIs and UTIs respectively were included. Bacterial identification and antibiotic susceptibility testing (Minimum Inhibitory Concentration- MIC based) were performed using Vitek 2 (bioMerieux Inc., Durham, NC). The Vitek panel preparation had included QC strains as recommended by Clinical \& Laboratory Standards Institute (CLSI) guidelines. The following antibiotics were included in the susceptibility testing ampicillin (A), ciprofloxacin (Cf), clindamycin (Cd), doxycycline (Do), erythromycin (E), levofloxacin (Lf), linezolid (Lz), moxifloxacin (Mf), nitrofurantoin $(\mathrm{Nf})$, tetracycline $(\mathrm{T})$, tigecycline $(\mathrm{Tg})$, vancomycin (Va), Oxacillin (Ox) amoxicillin/clavulanate (AC), Cefoxitin $(C n)$, ceftriaxone $(C i)$, gentamicin $(G)$, Tigecycline (Tg), aztreonam (Az), cefepime (Cp), ceftazidime ( $\mathrm{Ca})$, colistin $(\mathrm{Cl})$, imipenem (Im), meropenem $(\mathrm{Mm})$, minocycline $(\mathrm{Mc})$, pefloxacin (Pf), piperacillin (Pc), piperacillin/tazobactam (PT), rifampin (Rf), ticarcillin (Tc), tobramycin (Tb), trimethoprim/sulfamethoxazole (TS) and amikacin (Ak).

\section{Statistical analysis of data}

Data on the prevalence of drug resistance in bacterial pathogens causing BSIs and UTIs in patients admitted to ICUs was obtained and statistically analyzed using GraphPad prism 7.0.

\section{RESULTS}

Totally 79 patients with either BSI or UTI, or both, were admitted in ICU; during the years 2016 and 2017 were included. From the blood and/or urine of these patients, one or more bacterial strains were cultured.

Distribution of BSI and UTI among patients in ICU

A total 111 bacterial cultures were obtained from blood or urine of the 79 patients 
admitted in the ICU. Of 111 bacterial cultures, 73 (65.8\%) were from urine (UTIs) and 38 (34.2\%) were from blood (BSIs).

Statistical significance between the BSIs and UTIs in $\mathbf{2 0 1 7}$ when compared to 2016

There was a decrease in both BSIs and UTIs in 2017 when compared to 2016. By using Fisher's exact test, it was found that the twotailed $P$ value is less than 0.0001 . The association between infections (BSIs and UTIS) and years $(2016,2017)$ is considered extremely statistically significant.

Distribution of bacterial pathogens among BSIs

Totally 38 bacterial strains were isolated and identified from blood, of patients admitted in the ICU, by the Vitek 2 (bioMérieux Inc., Durham, NC) system. Among the Gram-positive bacteria,
13 isolates of coagulase negative Staphylococcus, 2 isolates of Enterococcus faecalis and one each of Staphylococcus aureus, Enterococcus faecium, and Leuconostoc citreum were obtained. Among Gram-negative bacteria, 10 isolates of Klebsiella pneumoniae, 2 isolates of $E$. coli and one each of Pseudomonas aeruginosa and Klebsiella oxytoca were obtained. Six isolates of Candida spp. were also obtained (Fig. 1).

Distribution of bacterial pathogens among UTIs

A total of 73 bacterial strains were isolated and identified from urine, of patients admitted in the ICU, by the Vitek 2 (bioMerieux Inc., Durham, NC) system. Among Gram negative bacteria, 16 isolates of Acinetobacter baumannii, 14 isolates of E. coli, 14 isolates of Klebsiella pneumoniae, 12 isolates of Pseudomonas aeruginosa, 3 isolates of

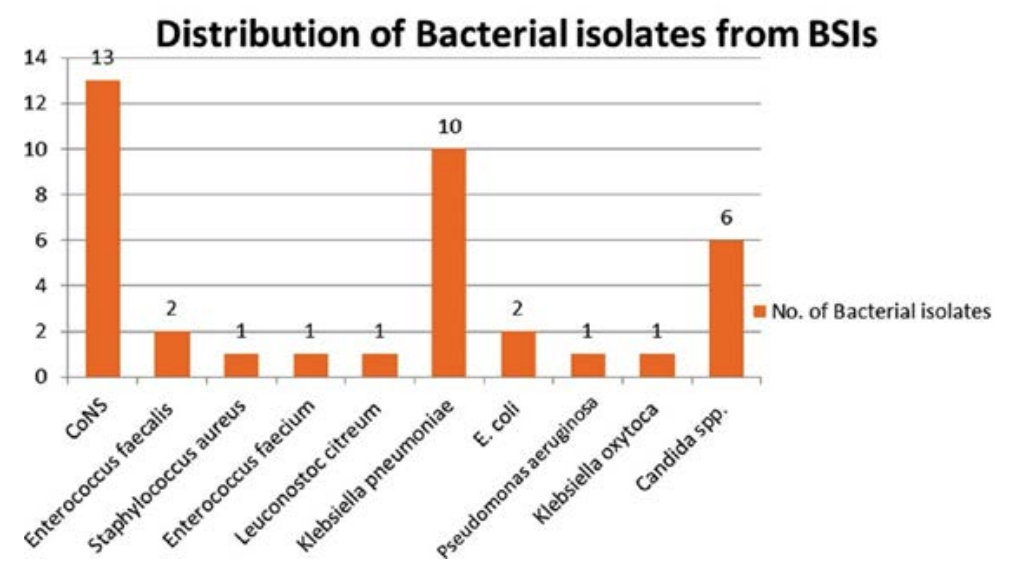

Fig. 1. Distribution of bacterial pathogens among BSIs in patients admitted to ICUs in 2016 \& 2017

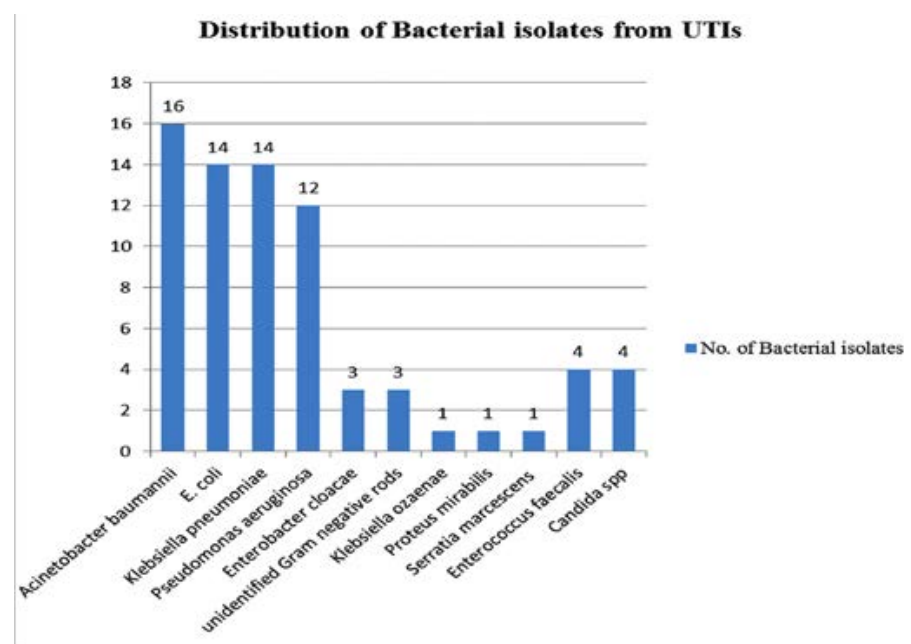

Fig. 2. Distribution of bacterial pathogens among UTIs in patients admitted to ICUs in 2016 \& 2017 


\section{Resistance profile of Klebsiella pneumoniae (prominent pathogen) isolated from blood of ICU patients in 2016-17}

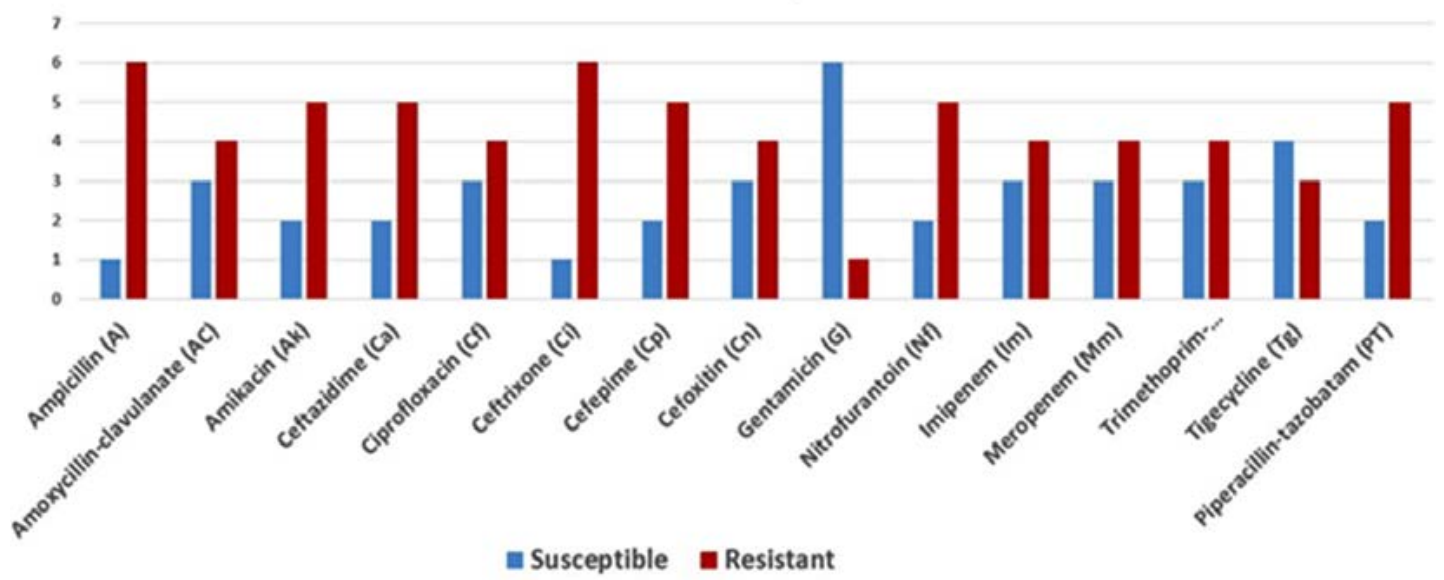

Fig. 3. Antimicrobial susceptibility trend of Klebsiella pneumoniae cultured from blood of ICU patients (2016-2017)

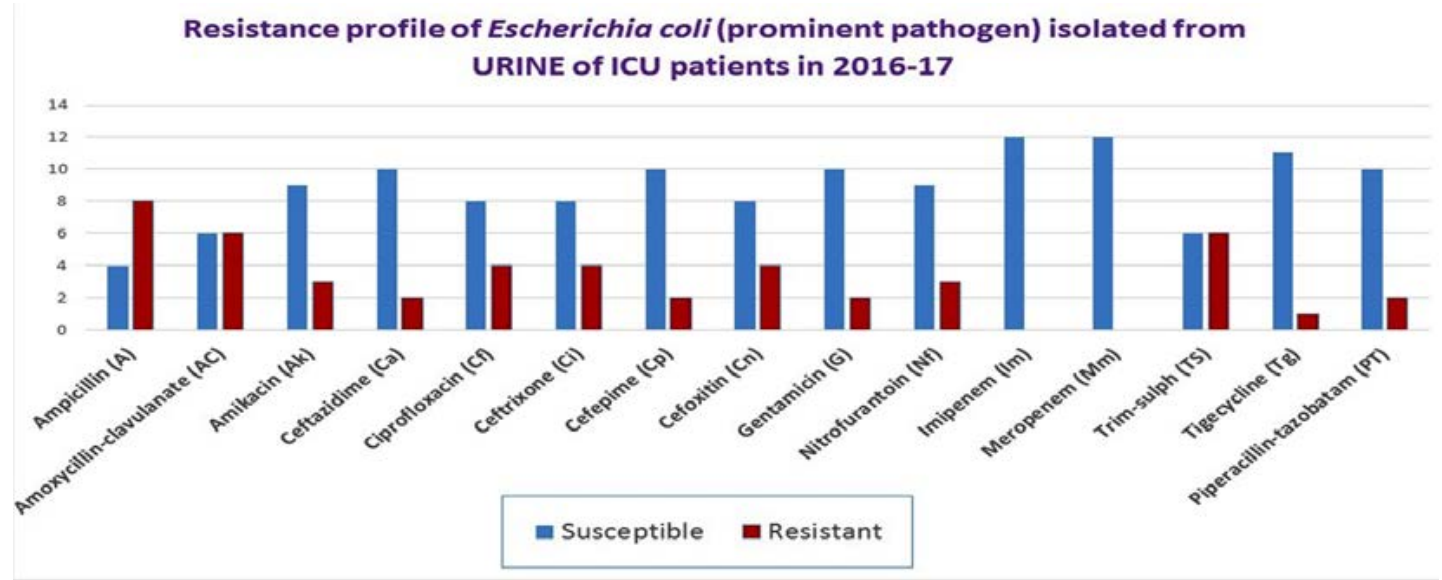

Fig. 4. Antimicrobial susceptibility trend of Escherichia coli cultured from urine of ICU patients (2016-2017)

Resistance profile of Pseudomonas aeruginosa (prominent pathogen) isolated from URINE of ICU patients in 2016-17

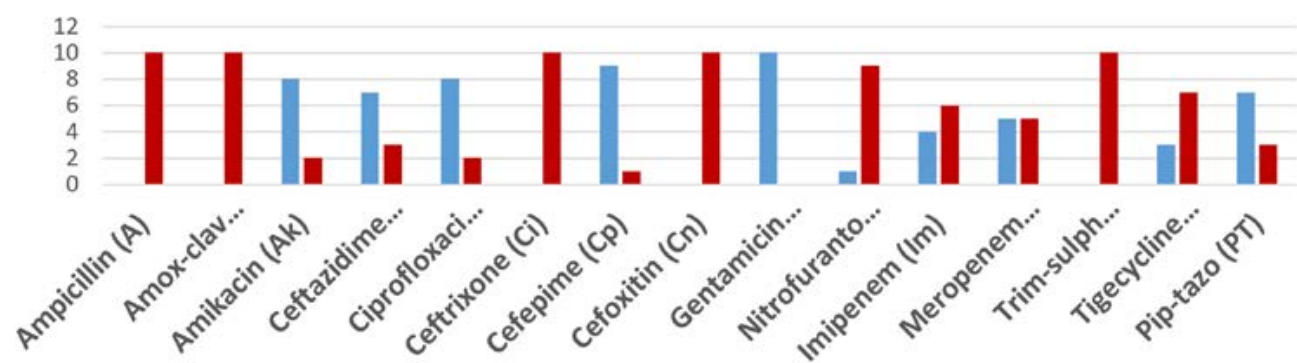

\section{- Susceptible $\mathbf{\text { Resistant }}$}

Fig. 5. Antimicrobial susceptibility trend of Pseudomonas aeruginosa cultured from urine of ICU patients (2016-2017) 
Enterobacter cloacae, 3 isolates of unidentified Gram negative rods, and one each of Klebsiella ozaenae, Proteus mirabilis, Serratia marcescens were obtained. Among Gram positive bacteria 4 isolates of Enterococcus faecalis and 4 isolates of Candida spp. were obtained (Fig. 2).

Antimicrobial susceptibility pattern of isolates cultured from blood of ICU patients

Among Klebsiella pneumoniae, the resistance rate of $85.7 \%$ was observed against ampicillin and ceftriaxone. The isolates of Klebsiella pneumoniae were uniformly resistant
(50 - 75\% resistant) to range of antibiotics tested amikacin, ceftazidime, ciprofloxacin, cefepime, nitrofurantoin, imipenem, meropenem and $\beta$-lactam plus $\beta$-lactamase inhibitor combinations. Interestingly, $85.7 \%$ Klebsiella pneumoniae were susceptible to a conventional antibiotic, gentamicin. It was alarming to notice $42.9 \%$ resistance to tigecycline (Fig. 3 and Table 1).

Antimicrobial susceptibility trend of isolates cultured from urine of ICU patients

Escherichia coli isolated from urine were $100 \%$ susceptible to carbapenems (imipenem and

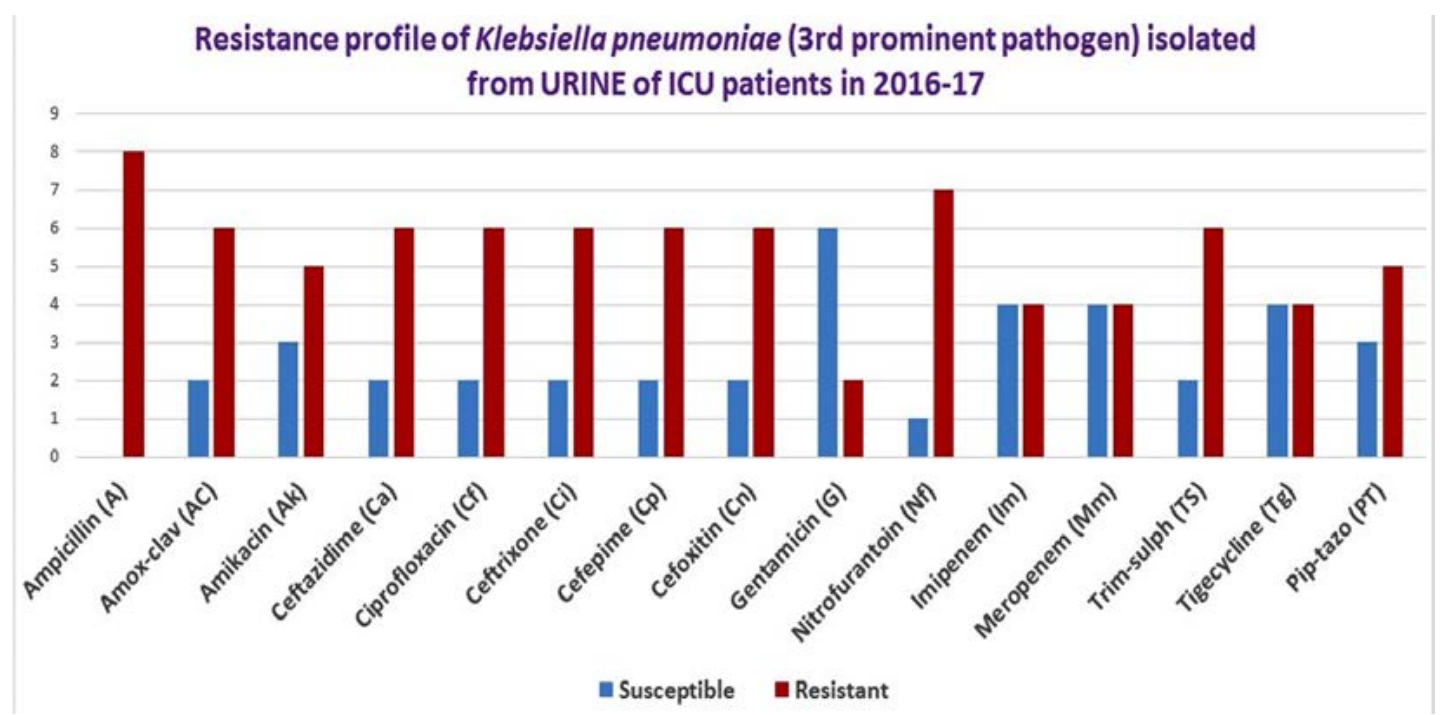

Fig. 6. Antimicrobial susceptibility trend of Klebsiella pneumoniae cultured from urine of ICU patients (2016-2017)

Resistance profile of Acinetobacter baumannii C. (4th prominent pathogen) isolated from URINE of ICU patients in 2016-17

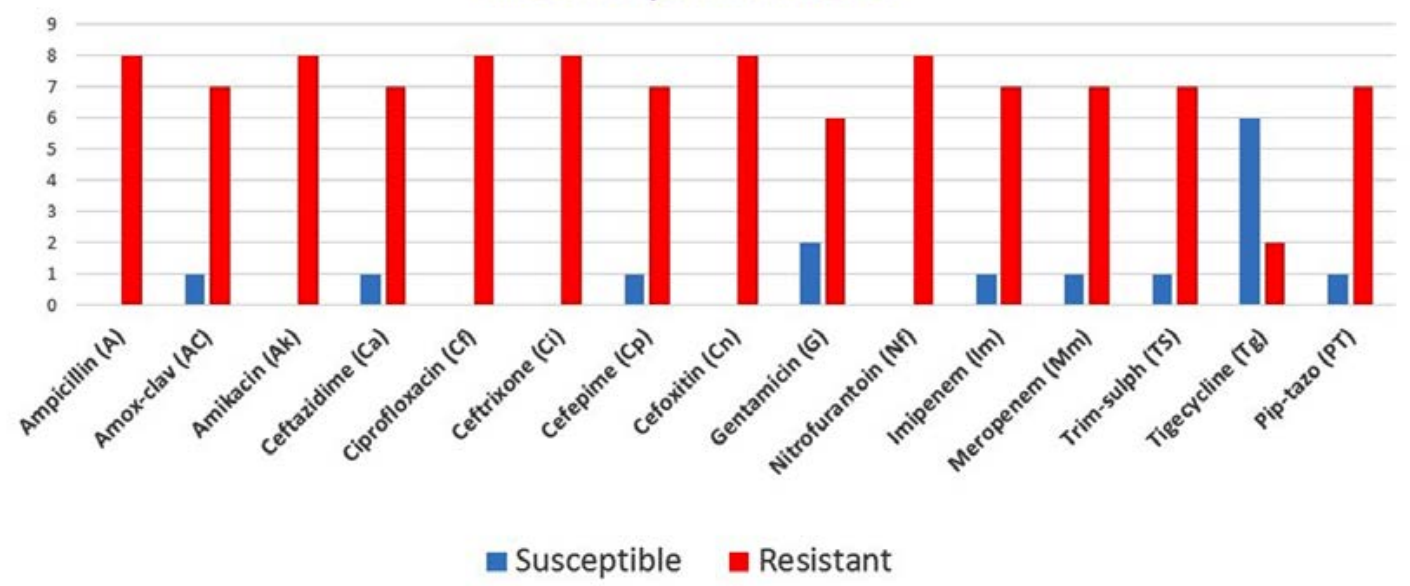

Fig. 7. Antimicrobial susceptibility trend of Acinetobacter baumannii complex cultured from urine of ICU patients (2016-2017) 


\section{Do I Need to Give Antibiotics?}
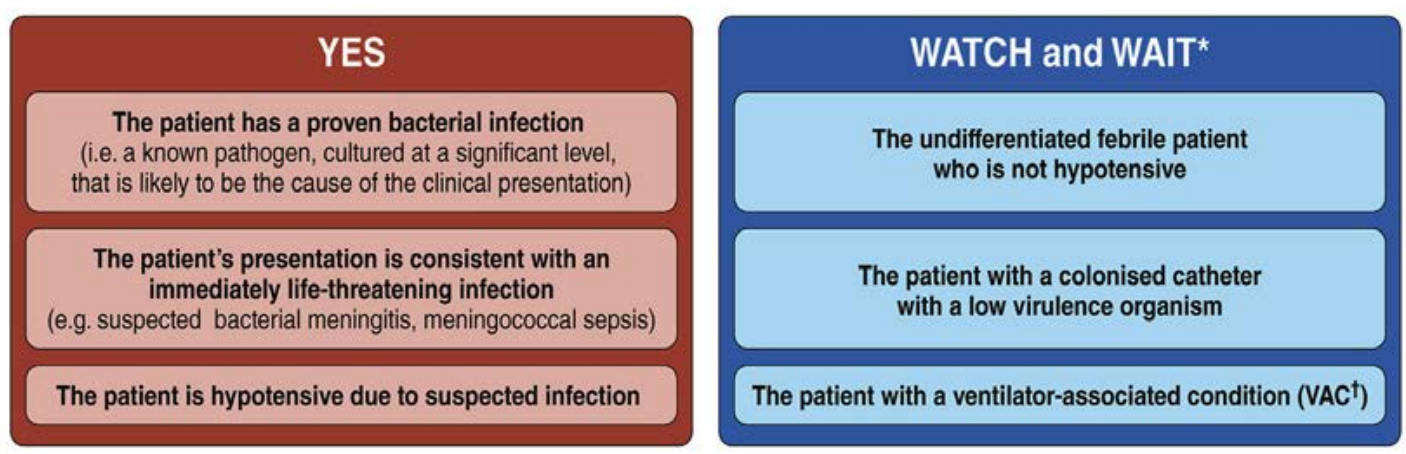

"Watch and Wait: Close monitoring of the patient in an high-dependency or intensive care setting for signs of deterioration whilst further investigations and attempts at source identification and control are carried out.

tVAC: An increase in the minimum PEEP during a 24-hour period of $3 \mathrm{~cm} \mathrm{H}_{2} \mathrm{O}$ or an increase in the minimum $\mathrm{FiO}_{2}$ during a 24 hour period of $20 \%$, after a period of 48 hours of stable ventilator settings

Fig. 8. Do I need to give antibiotics?- Summary ${ }^{1}$.

carbapenem). Isolates ranged $50-75 \%$ in resistance to array of antibiotics tested. Of the isolates, 91.75\% were susceptible to tigecycline (Fig. 4 and Table 2).

Pseudomonas aeruginosa isolates cultured from urine of ICU patients were $100 \%$ resistant to ampicillin, amoxicillin-clavulanate, ceftriaxone, cefoxitin, and trimethoprimsulphamethoxazole. Interestingly, $100 \%$ isolates were susceptible to gentamicin and $90 \%$ were susceptible to cefepime and nitrofurantoin. For other range of antibiotics tested, the resistance rate varied from $50 \%$ to $75 \%$ (Fig. 5 and Table 2).

Klebsiella pneumoniae isolates cultured from urine of ICU patients uniformly displayed $75 \%$ resistance to ciprofloxacin, trimethoprimsulphamethoxazole, ceftazidime, ceftriaxone, cefoxitin, cefepime, amoxiclave and piperacillintazobactam. Overall, $87.5 \%$ of $K$. pneumoniae were resistant to nitrofurantoin. Further, $75 \%$ of K. pneumoniae were susceptible to gentamicin. (Fig. 6 and Table 2).

Acinetobacter baumannii complex cultured from urine of ICU patients displayed an alarmingly high rate of consistent resistance to all the antibiotics tested. Further, up to $50 \%$ of the isolates were susceptible to gentamicin and up to $75 \%$ of the isolates were susceptible to tigecycline (Fig. 7 and Table 2).

\section{DISCUSSION}

Infection surveillance is vital for quality care, prevention, and suitable management of ICU-acquired infections. Occurrence of ICUacquired infections vary in different settings - ICU types and type of population ${ }^{13}$. Knowledge about prominent pathogens in ICU acquired infections and their resistance profile can help us frame specific infection control policies and avoid spread of such infections.

Rosental et al., in their multi centric study demonstrated the following prevalent type of infections - ventilator-associated pneumonia $(41 \%)$, BSIs $(30 \%)$ and urinary tract infections $(29 \%)^{14}$. In the present study, a total 111 bacterial cultures were obtained from blood or urine of the 79 patients admitted in the ICU. Of 111 bacterial cultures, 73 (65.8\%) were from urine (UTIs) and 38 (34.2\%) were from blood (BSIs) Habibi et al., in a study on nosocomial infections found UTI in $24 \%$, and BSI in $24 \%$ subjects $^{15}$. United States National Nosocomial Infections Surveillance data showed UTIs in $23 \%$ and BSIs in $14 \%$ subjects $^{16}$. Kamat et al., showed UTI (26.6\%) to be the most common infection in ICU set up ${ }^{17}$.

Several studies have observed that Gram-negative pathogens are the main cause of nosocomial infections, which is in concordance with our study ${ }^{16,17}$. In our study, among the isolates 


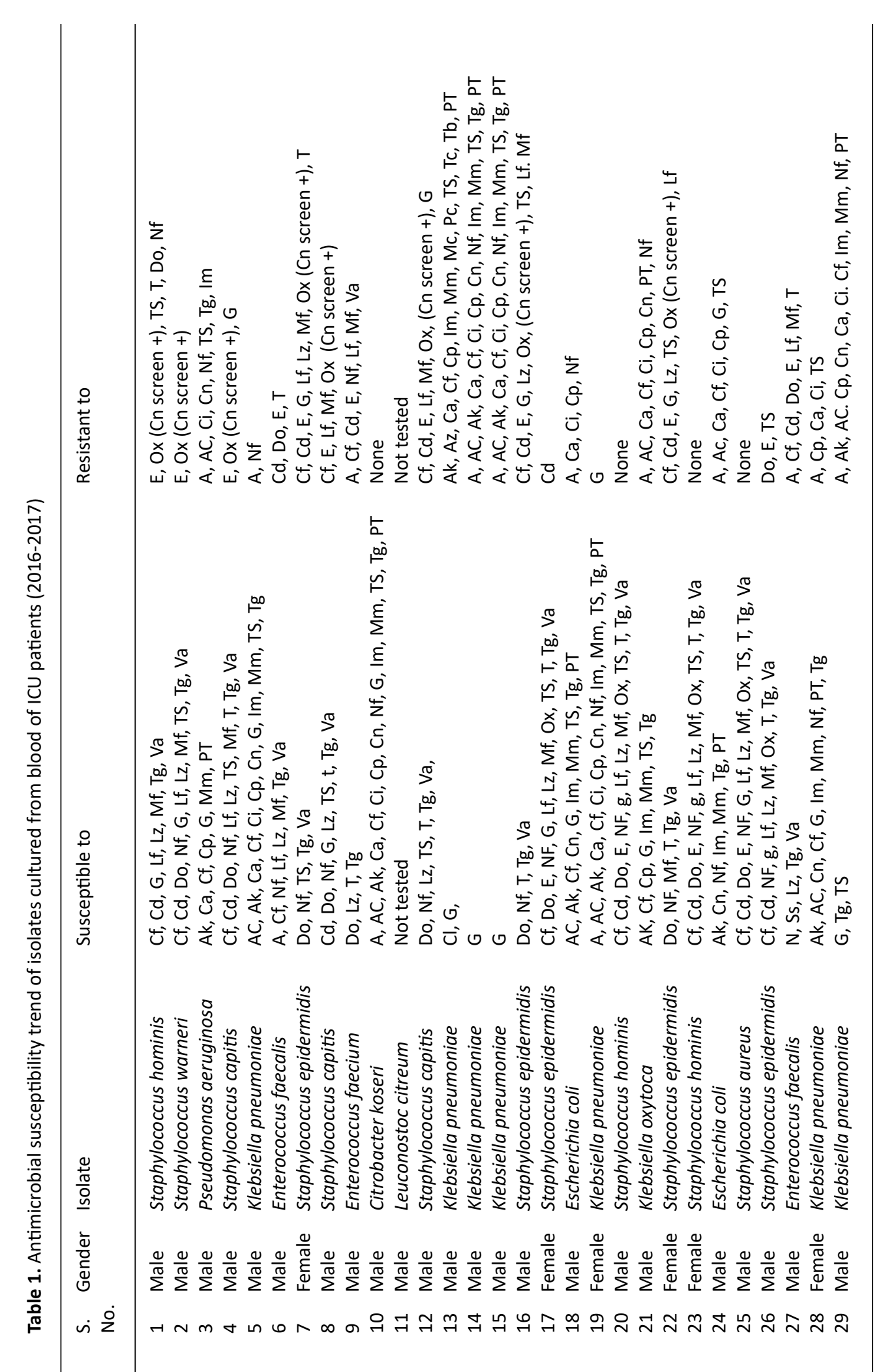




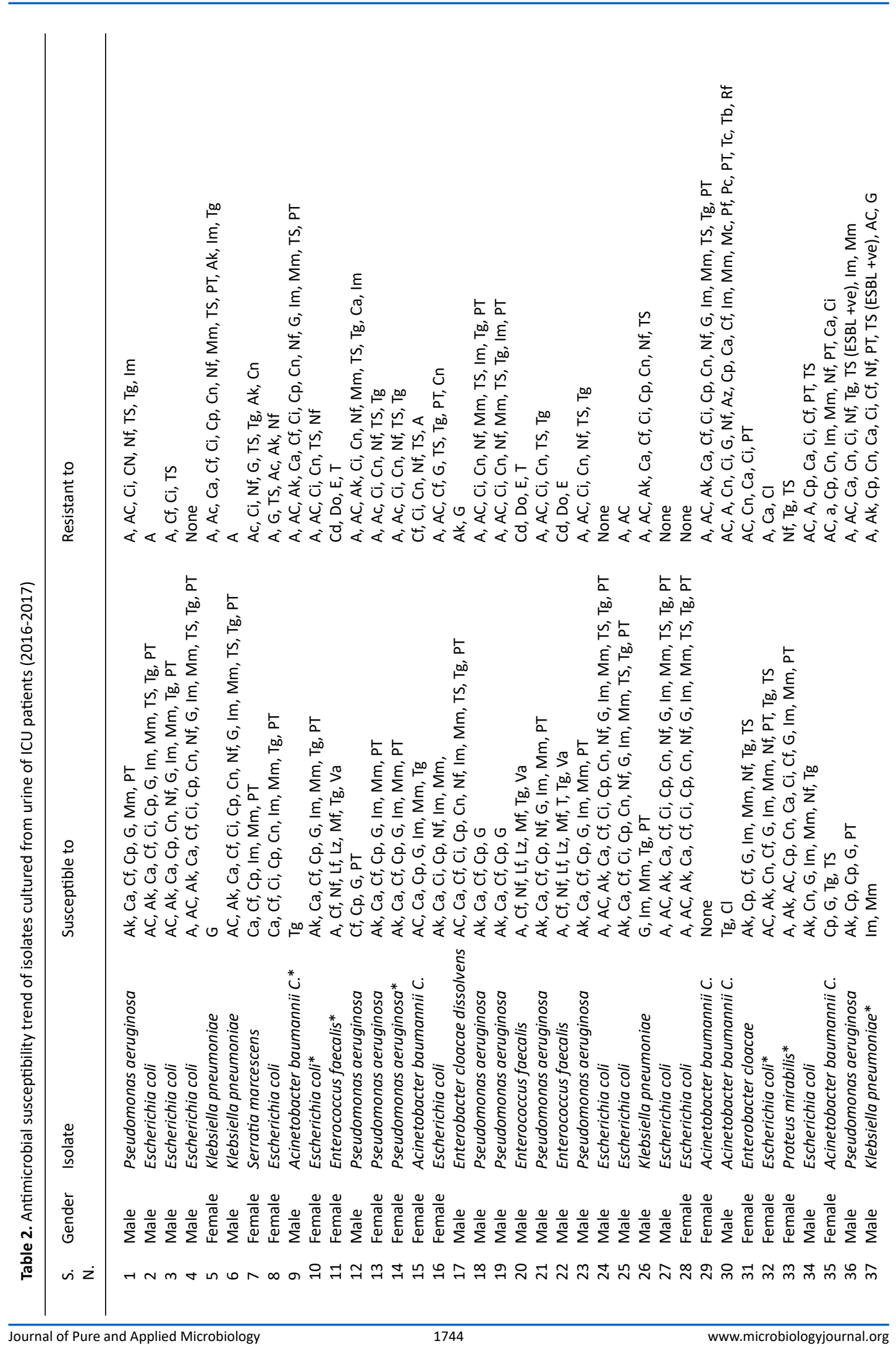




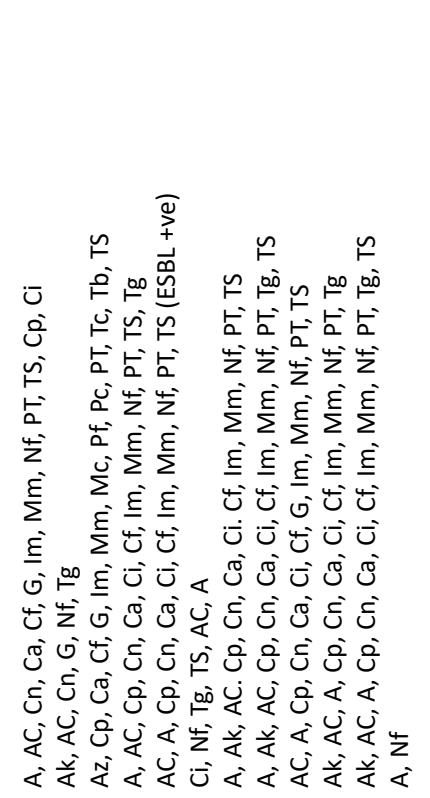

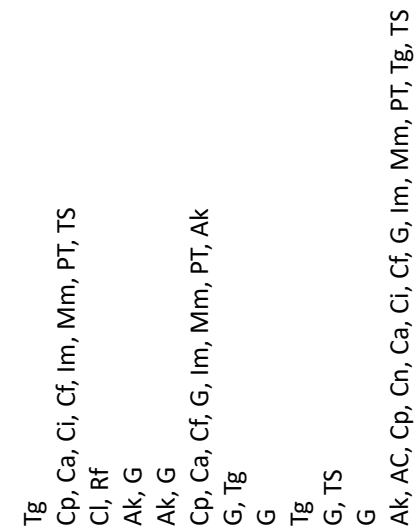

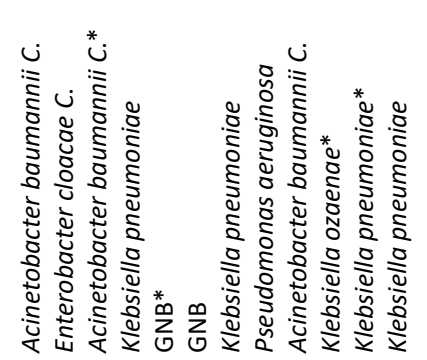

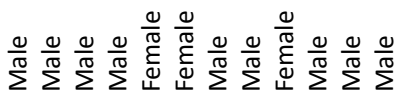

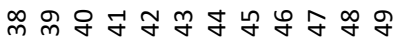

cultured from blood of ICU admitted patients, Enterobacteriaceae (36.8\%) was the most common pathogen. Coagulase negative Staphylococci (CoNS) among Gram-positive bacteria were cultured from $34.2 \%$ of the subjects. Recent research studies have indicated Pseudomonas spp., Acinetobacter spp., Escherichia coli and Klebsiella spp. to be the most common pathogens in ICU-acquired infections ${ }^{15,17}$.

Ceftazidime and aztreonam are loosing their activity against the Gram-negative microorganisms. The fourth generation cephalosporins have an intrinsic high activity against the inducible Enterobacteriaceae. The introduction of cefepime for nosocomial infections led to a remarkable drop in the number of Enterobacter isolates combined with important decreases in Enterobacter resistance towards several antibiotics. Prescribing antibiotics is therefore an important task. Concentrating sick people in crowded spaces leads to an increase in the incidence of infections ${ }^{18}$.

Infections acquired in the ICU are associated with microbiological isolates different from those acquired in the community, and are often associated with resistant organisms. Further evidence suggested an algorithm regarding antibiotic initiation for patients in the ICU (Fig. 8) ${ }^{1}$.

The UAE AMR National Surveillance Report stated that, $E$. coli was the most common pathogen of BSIs followed by K. pneumoniae and $S$. aureus. However, their study showed that $K$. pneumoniae showed a resistance of $37 \%$ against ceftriaxone and $12 \%$ against carbapenems ${ }^{2}$.

From the Global Antimicrobial Surveillance System, World Health Organization (GLASS-WHO) study, E. coli is the dominant agent causing UTIs and $K$. pneumoniae is the leading cause of BSIs followed by $E$. coli. The common finding between our study and GLASS-WHO is that, E. coli displays the highest resistance to Ampicillin. In addition, the resistance is very low for carbapenems namely, imipenem and meropenem ${ }^{3}$.

In support to our study, their study found that $K$. pneumoniae from urine samples displays high resistance to ceftriaxone in blood and urine. In addition, the agent shows equal proportion of resistance to ceftazidime, cefotaxime and cotrimoxazole. Lastly, the resistance profile is very 
low vis-a-vis urine cultures against carbapenems, (imipenem and meropenem) ${ }^{3}$.

In our study, among the isolates cultured from urine of ICU admitted patients, Enterobacteriaceae (46.6\%) was the most common pathogen. Non-fermenter Gram-negative bacteria were present at $38.4 \%$. Enterococcus faecalis was the only Gram-positive pathogen cultured from urine.

Venkataraman et al., found isolates of Klebsiella spp., E. coli, Acinetobacter spp., and Pseudomonas spp., to be resistant to the 3rd generation cephalosporins. They found around $70 \%$ isolates to be resistant to all the antibiotics tested. They attributed this resistance to use of 3rd generation cephalosporins for already received empiric treatment, which induces antibiotic resistance ${ }^{15,19}$.

Of late, in all hospital settings including ICUs the antibiotic resistance has escalated worldwide. This is responsible for inappropriate and delayed prescription that further leads to high mortality rates ${ }^{20}$. Tumbarello et al., stated BSIs caused by extended-spectrum $\beta$-lactamase (ESBL)producers leads to 3-fold increase in mortality ${ }^{21}$. In our study, among $K$. pneumoniae isolated from blood a resistance rate of $85.7 \%$ was observed against ampicillin and ceftriaxone. The isolates of K. pneumoniae were uniformly resistant (50-75\% resistant) to range of antibiotics tested, including ciprofloxacin, trimethoprim-sulphamethoxazole amikacin, cefoxitin, ceftazidime, ceftriaxone, cefepime, nitrofurantoin, imipenem, meropenem and $\beta$-lactam plus $\beta$-lactamase inhibitor combinations (amoxiclave and piperacillintazobactam).

Interestingly, $85.7 \%$ K. pneumoniae were susceptible to a conventional antibiotic, gentamicin. It was alarming to notice $42.9 \%$ resistance to tigecycline.

E. coli isolated from urine were $100 \%$ susceptible to carbapenems (imipenem and carbapenem). Isolates ranged $50-75 \%$ in resistance to array of antibiotics tested. Of the isolates, 91.75\% were susceptible to tigecycline.

$P$. aeruginosa isolates cultured from urine of ICU patients were $100 \%$ resistant to ampicillin, amoxicillin-clavulanate, ceftriaxone, cefoxitin, and trimethoprim-sulphamethoxazole. Interestingly,
$100 \%$ isolates were susceptible to gentamicin; and $90 \%$ susceptible to cefepime and nitrofurantoin. For other range of antibiotics tested, the resistance rate varied from $50 \%$ to $75 \%$.

K. pneumoniae isolates cultured from urine of ICU patients uniformly displayed $75 \%$ resistance to ciprofloxacin, ceftriaxone, cefoxitin and cefepime. Overall, $87.5 \%$ of $K$. pneumoniae were resistant to nitrofurantoin. Further, $75 \%$ of K. pneumoniae were susceptible to gentamicin. A. baumannii complex cultured from urine of ICU patients displayed an alarmingly high rate of consistent resistance to all the antibiotics tested. Further, the isolates were $50-75 \%$ susceptible only to selective antibiotics such as, gentamicin, imipenem, meropebnem, and tigecycline.

The EUROBACT study demonstrated the role of MDR Gram-negative pathogens in more than $50 \%$ cases in ICUs ${ }^{22}$. BSIs caused by ESBLs is a major challenge for clinicians. The randomized clinical trials have not demonstrated the effectiveness of $\beta$-lactam $/ \beta$-lactamase inhibitor combinations (such as piperacillintazobactam). In critically ill patients, carbapenems are used as the first drug of choice ${ }^{23}$. Due to this overuse and misuse, antibacterial resistance to carbapenem has gradually increased. Carbapenem resistance in Europe, among hospital acquired BSI causing pathogens, such as, Acinetobacter spp., K. pneumoniae and Pseudomonas spp. has been shown to be around $69 \%, 37 \%$ and $5.7 \%$ respectively ${ }^{22}$. Very few options for treating carbapenem-resistant pathogens are available now. The use of combination antibiotics including tigecycline or colistin along with a carbapenem have been preferred due to its benefit over observational monotherapy studies ${ }^{21}$. Unfortunately, pathogens, which are multi-drug resistant (MDR), are often resistant to many other antibiotics such as aminoglycosides and fluoroquinolones. Further, colistin resistance has become common among MDR Gram-negative pathogens ${ }^{24}$.

Results from a single-center study varies compared to multicenter study, since the patients from a single hospital may have different risk factors, severity of infection, and use of invasive devises ${ }^{16}$. Length of stay in hospital is an important reason for the development of infection ${ }^{15}$. Longer 
stay necessitates longer period of insertion of devices and chances of colonization with MDR pathogens.

\section{CONCLUSION}

Coliforms (K. pneumoniae in BSI; E. coli in UTI) happen to be the prominent pathogens in our ICU admitted patients. K. pneumoniae isolated from blood demonstrated $50-75 \%$ resistance to a range of antibiotics tested - amikacin, ceftazidime, ciprofloxacin, cefepime, nitrofurantoin, imipenem, meropenem and beta-lactam+beta-lactamase inhibitor combinations. It was alarming to notice $42.9 \%$ resistance to tigecycline among $K$. pneumoniae isolated from blood. K. pneumoniae isolates cultured from urine of ICU patients uniformly displayed $75 \%$ rate of resistance to ciprofloxacin, ceftriaxone, cefoxitin and cefepime. Overall, $87.5 \%$ of $K$. pneumoniae were resistant to nitrofurantoin. Interestingly, it is of relief to observe $85.7 \%$ Klebsiella pneumoniae isolated from blood and $75 \% \mathrm{~K}$. pneumoniae isolated from urine being susceptible to a conventional antibiotic, gentamicin. E. coli isolated from urine were $100 \%$ susceptible to carbapenems (imipenem and carbapenem) and $91.75 \%$ susceptible to tigecycline. Overall, $90 \%$ of $P$. aeruginosa were susceptible to nitrofurantoin. Overall, a resistance rate of $50-75 \%$ was observed against the range of antibiotics. The rapid spread of these MDR pathogens demands for national and regional guidelines. Antimicrobial guidelines for empirical treatment and to treat infections in ICUs should be designed based on local microbiological data and resistance profiles of pathogens. Infection prevention \& control (IPC) measures should be in place for reducing the spread of antimicrobial resistant pathogens. These measures can prevent further infections and AMR spread.

\section{ACKNOWLEDGMENTS}

We would like to acknowledge the Central Research Laboratory (CRL) facility of RAK Medical \& Health Sciences University (RAKMHSU), Ras Al Khaimah, UAE.

\section{CONFLICT OF INTEREST}

The authors declare that there is no conflict of interest.

\section{AUTHORS' CONTRIBUTION}

All authors listed have made a substantial, direct and intellectual contribution to the work, and approved it for publication.

\section{FUNDING}

None.

\section{ETHICS STATEMENT}

This study was approved by RAKMHSUREC and RAK-REC committees.

\section{DATA AVAILABILITY}

All datasets generated or analyzed during this study are included in the manuscript.

\section{REFERENCES}

1. Denny KJ, Wale J De, Laupland KB, Harris PNA, Lipman J. When not to start antibiotics: avoiding antibiotic overuse in the intensive care unit. Clin Microbiol Infect. 2020;26:35-40. doi: 10.1016/j.cmi.2019.07.007

2. Thomsen J, Abdulrazzaq N. United Arab Emirates national surveillance system for antimicrobial resistance, 2019.

https://www.escmid.org/escmid_publications/ escmid_elibrary/material/?mid=67722.

3. World Health Organization. Global Antimicrobial Resistance Surveillance System (GLASS). 2019;216-219.

4. Mnatzaganian G, Galai N, Sprung CL, et al. Increased risk of bloodstream and urinary infections in intensive care unit (ICU) patients compared with patients fitting ICU admission criteria treated in regular wards. $J$ Hosp Infect. 2005;59(4):331-342. doi: 10.1016/j. jhin.2004.07.028

5. Bassetti M, Righi E, Alessia Carnelutti. Bloodstream infections in the Intensive Care Unit, Virulence. 2016;7:267-279. doi: 10.1080/21505594.2015.1134072

6. Nasa P, Juneja D, Singh O, et al. Incidence of bacteremia at the time of ICU admission and its impact on outcome. Indian J Anaesth. 2011;55(6):594-598. doi: 10.4103/0019-5049.90615

7. Ammerlaan HSM, Harbarth S, Buiting AGM, et al. Secular trends in nosocomial bloodstream infections: antibiotic-resistant bacteria increase the total burden of infection. Clinical Infect Dis. 2013;56(6):798-805. doi: $10.1093 /$ cid/cis1006

8. Magiorakos A-P, Srinivasan A, Carey $R$, et al. Multidrug-resistant, extensively drug-resistant and pandrug-resistant bacteria: an international expert proposal for interim standard definitions for acquired resistance. Clin Microbiol Infect. 2012;18(3):268-281. doi: 10.1111/j.1469-0691.2011.03570.x

9. Carlet J, Collignon P, Goldmann D, et al. Society's failure to protect a precious resource: antibiotics. Lancet. 2011;378(9788):369-371. doi: 10.1016/S01406736(11)60401-7

10. Russotto V, Cortegiani A, Graziano G, et al. Bloodstream 
infections in intensive care unit patients: distribution and antibiotic resistance of bacteria. Infect Drug Resist. 2015;8:287-296. doi: 10.2147/IDR.S48810

11. Jaggi N, Sissodia P. Multimodal supervision programme to reduce catheter associated urinary tract infections and its analysis to enable focus on labour and cost effective infection control measures in a tertiary care hospital in India. J Clin Diagn Res. 2012;6(8):13721376. doi: 10.7860/JCDR/2012/4229.2362

12. Silver SA, Baillie L, Simor AE. Positive urine cultures: A major cause of inappropriate antimicrobial use in hospitals. Can J Infect Dis Med Microbiol. 2009;20:107111. doi: $10.1155 / 2009 / 702545$

13. Gastmeier P, Sohr D, Just HM, et al. How to survey nosocomial infections. Infect Control Hosp Epidemiol. 2000;21(6):366-370. doi: 10.1086/501774

14. Rosenthal VD, Maki DG, Salomao R, et al. Deviceassociated nosocomial infections in 55 Intensive Care Units of 8 developing countries. Ann Intern Med. 2006;145(8):582-591. doi: 10.7326/0003-4819-1458-200610170-00007

15. Habibi S, Wig N, Agarwal S, et al. Epidemiology of nosocomial infections in medicine intensive care unit at a tertiary care hospital in Northern India. Trop Doct. 2008;38:233-235. doi: 10.1258/td.2008.070395

16. Richards MJ, Edwards JR, Culver DH, et al. Nosocomial infections in combined medical-surgical Intensive Care Units in the United States. Infect Control Hosp Epidemiol. 2000;21(8):510-515. doi: 10.1086/501795

17. Kamat US, Ferreira AMA, Savio R, Motghare DD. Antimicrobial resistance among nosocomial isolates in a teaching hospital in Goa. Indian J Community Med. 2008;33(2):89-92. doi: 10.4103/0970-0218.40875

18. Demy $\mathrm{HE}$, Jansens $\mathrm{H}$, Laer $\mathrm{FV}$, leven $\mathrm{M}$, Goossens $\mathrm{H}$, Bossaert LL. Strategies for selecting antibiotics in intensive care units. Clin Microbiol Infect.
1999;5(S1):S29-S34. doi: 10.1111/j.1469-0691.1999. tb00722.x

19. Venkataraman R, Divatia JV, Ramakrishnan N, et al. Multicenter observational study to evaluate epidemiology and resistance patterns of common intensive care unit-infections. Indian J Crit Care Med. 2018;22:20-26. doi: 10.4103/ijccm.IJCCM_394_17

20. Ammerlaan HSM, Harbarth S, Buiting AGM, et al. Secular trends in nosocomial bloodstream infections: Antibiotic-resistant bacteria increase the total burden of infection. Clin Infect Dis. 2013;56:798-805. doi: 10.1093/cid/cis1006

21. Tumbarello $M$, Sanguinetti $M$, Montuori $E$, et al. Predictors of mortality in patients with bloodstream infections caused by extended-spectrum-betalactamase-producing Enterobacteriaceae: Importance of inadequate initial antimicrobial treatment. Antimicrob Agents Chemother. 2007;51:1987-1994. doi: 10.1128/AAC.01509-06

22. Tabah A, Koulenti D, Laupland K, et al. Characteristics and determinants of outcome of hospital-acquired bloodstream infections in intensive care units: The EUROBACT International Cohort Study. Intensive Care Med. 2012;38:1930-1945. doi: 10.1007/s00134-0122695-9

23. Harris PN, Tambyah PA, Paterson DL. $\beta$-lactam and $\beta$-lactamase inhibitor combinations in the treatment of extended-spectrum $\beta$-lactamase producing Enterobacteriaceae: Time for a reappraisal in the era of few antibiotic options? Lancet Infect Dis. 2015;15(4):475-485. doi: 10.1016/S14733099(14)70950-8

24. Bassetti M, Righi E. SDD and colistin resistance: End of a dream? Intensive Care Med. 2014;40:1066-1067. doi: 10.1007/s00134-014-3328-2 